\title{
Yes, I BeLIEVE IN THE CBR!
}

\author{
Gilberto Friedman ${ }^{1,2}$ \\ Editor-in-Chief
}

Two years ago, the Clinical and Biomedical Research entered a new era. I am honored to be the second Editor-in-Chief of what has become known as the 'CBR Journal'. I aim to follow Alexandre Zavascki's successes and his hard work and accomplishments as the first Editor-in-Chief. Those of you who remember the former Revista do Hospital de Clínicas de Porto Alegre have seen many changes in the last 2 years. The number of articles has now risen to around 65 articles annually and the Journal received an increased number of papers for consideration.

The Journal has also changed to a complete online version, we are saving trees! Now entering its 3rd year, the Journal has plans to fly high. This will be possible due to the combined efforts of a strong and renewed Editorial Board with a range of specialist expertise in all areas of health care, a dedicated Editorial Office staff, and the team of volunteers that are giving their time to act as peer reviewers for the CBR. The Journal restructured its Editorial Board to comprise a team of Editors to evaluate submissions of some specific areas of health care or medical science. We are looking for ways to rapidly and fairly select the best articles for publication.

All changes in the Journal were thoroughly considered to meet the Journal's scope and aims, and to please our readers and authors.

On behalf of the Journal, I thank Eduardo Passos as the Coordinator of the Grupo de Pesquisa e Pós-Graduação for the invitation and Alexandre's continued support to help conduct the Journal through the next years. Yes, I believe in the CBR!
Clin Biomed Res. 2016;36(1):3

1 Departamento de Medicina Interna, Faculdade de Medicina, Universidade Federal do Rio Grande do Sul (UFRGS). Porto Alegre, RS, Brazil.

2 Serviço de Medicina Intensiva, Hospital de Clínicas de Porto Alegre Porto Alegre, RS, Brazil.

Corresponding author: Gilberto Friedman gfriedman@hcpa.edu.br Serviço de Medicina Intensiva, Hospital de Clínicas de Porto Alegre, Rua Ramiro Barcelos, 2350. 90035-903, Porto Alegre, RS, Brazil. 